\title{
Control of the Interfacial Instabilities in a circular Hele-Shaw cell oscillating with a periodic angular velocity
}

\author{
J. BOUCHGL ${ }^{1,2}$, M. SOUHAR ${ }^{3}$ \\ 1. Institut Supérieur des Pêches Maritimes - Agadir (jamila.bouchgl@gmail.com) \\ 2. Laboratory of energy Engineering, Materials and Systems, ENSA, Ibn Zohr University, Agadir, Morocco. \\ 3. LEMTA, UMR CNRS 7563, université de lorraine, France.
}

\begin{abstract}
:
The stability of an interface of two viscous immiscible fluids of different densities and confined in a Hele-Shaw cell which is oscillating with periodic angular velocity is investigated. A linear stability analysis of the viscous and time-dependent basic flows, generated by a periodic rotation, leads to a time periodic oscillator describing the evolution of the interface amplitude. In this study, we examine mainly the effect of the frequency of the periodic rotation on the interfacial instability that occurs at the interface.
\end{abstract}

Keywords: Hele-Shaw cell, Parametric instability, periodic rotation, Stability analysis

\section{Introduction}

The interfacial instabilities in a rotating Hele-Shaw cell is the subject of several works [1-5]. These investigations have mainly focused on the understanding fingering mechanisms of the interface in the alternative configuration, where a denser inner fluid surrounded by another fluid of low density under constant rotational motion about an axis perpendicular to the cell plates. In this spirit, Schwartz [2] has performed the linear stability analysis of an initially concentric circular drop in the case of high density and viscosity contrast, water-air. In this work, the Coriolis forces are included in an ad hoc manner and it was shown that the circular drop is unstable both to translation and depending on the rotation rate, to a number of fingering modes. The linear stability analysis performed in [2] has been extended to arbitrary density and viscosity contrast by Carrillo et $a l$. [3-4]. Thereafter, Miranda [5] has determined the growth rate using a linear stability analysis and neglecting Coriolis forces in the presence of centrifugal forces and magnetic ones. He has showed that the magnetic forces act to stabilize the fluid interface. Later on, Abidate et al. [6] have reported analytical results and performed a linear stability analysis to study the influence of both Coriolis forces and curvature parameters on the linear growth rate. It was shown that the Coriolis forces does not alter the stability of a dis- turbance with a particular wave number but reduces the maximum growth rate.

Recently and in contrast to the majority of studies carried out in the rotating Hele-Shaw cell setup, the effect of time dependent angular velocity on the dynamics of such confined flows is only started to be investigated in the literature by Bouchgl et al. [7] and Anjos et al. [8]. In [7], the authors present a mathematical formulation of the physical problem based on the inviscid linear stability analysis. Hereafter, Anjos et al. [8] have focused on examining how the time-dependent angular velocity and the inertia affect the finger competition events that traditionally arise in rotating Hele-Shaw flows.

Following the study carried out on the modulation of rotation [7-8], we are interested in the present work to a zero mean modulation of the cell. In this context, we investigate the effect of frequency on the threshold of instability for the initially unstable configuration, where the inner fluid and the outer one are considered respectively heavy and light.

\section{Formulation}

\subsection{Governing equations}

Consider two viscous immiscible incompressible fluids confined in a rotating Hele-Shaw cell of thickness $e$ which is oscillating with the periodic angular velocity, $\boldsymbol{\Omega}^{*}\left(t^{*}\right)=$ $\left.\Omega_{m} \cos \left(\omega t^{*}\right)\right) \mathbf{k}$ where $\Omega_{m}$ and $\omega$ are respectively the amplitude and frequency of the modulated rotation (see Fig.1). Each fluid layer is characterized by the density $\rho_{j}$, the dynamic viscosity $\mu_{j}$ where the subscripts $j=1,2$ denotes the inner and outer layer respectively. Neglecting the Coriolis forces, the physical problem is governed by the following set of equations:

$$
\begin{aligned}
\nabla . \mathbf{V}_{j}^{*} & =0 \quad(\mathbf{j}=1,2) \\
\frac{d \mathbf{V}_{j}^{*}}{d t^{*}}= & -\frac{1}{\rho_{j}} \nabla p_{j}^{*}+\nu_{j} \Delta \mathbf{V}_{j}^{*}-\boldsymbol{\Omega}^{*}\left(t^{*}\right) \wedge\left(\boldsymbol{\Omega}^{*}\left(t^{*}\right) \wedge \mathbf{r}^{*}\right) \\
& -\frac{d \boldsymbol{\Omega}^{*}\left(t^{*}\right)}{d t^{*}} \wedge \mathbf{r}^{*}
\end{aligned}
$$


with $\frac{d}{d t^{*}}=\frac{\partial}{\partial t^{*}}+\mathbf{V}_{j}^{*} . \nabla$ is a total derivative, $p_{j}^{*}$ is the hydrodynamic pressure, $\mathbf{V}_{j}^{*}=\left(u_{j}^{*}, v_{j}^{*}\right)$ is the dimensional velocity in each fluid layer and $r^{*}$ the dimensional radial distance from the rotation axis. The values $z^{*}= \pm \frac{e}{2}$ correspond to the vertical boundaries of the cell. Moreover, the gravity is neglected considering that $g \ll R \Omega_{m}^{2}$

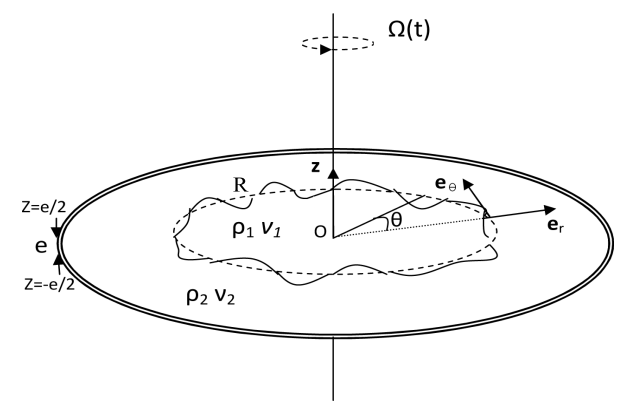

Figure 1: Sketch of the Hele-Shaw cell subject to a periodic angular velocity.

\subsection{Base flow solution}

Unlike in [8] where both inertia and time dependence of the angular velocity are not taking into account in the basic state, in this work, the term $\frac{d \boldsymbol{\Omega}^{*}\left(t^{*}\right)}{d t^{*}} \wedge \mathbf{r}^{*}$ produces a basic velocity field having an azimuth component $\left(0, V_{j}^{* b}(z, t)\right)$. This basic solution, $\left.V_{j}^{* b}(z, t)\right)$, satisfies the equation of continuity, $\mathrm{Eq}(1)$, and the equation of momentum, Eq.(2). Using the nonslip boundary conditions on the horizontal walls, $V_{j}^{* b}=0$ at $z^{*}= \pm \frac{e}{2}$, and with respect to the $z$ coordinate, the averaged velocity field and the pressure, in the rest state, are given by:

$$
\begin{aligned}
& \bar{V}_{j}^{* b}=r^{*}\left[\bar{F}_{j} \cos \left(\omega t^{*}\right)+\bar{G}_{j} \sin \left(\omega t^{*}\right)\right] \\
& \bar{P}_{j}^{* b}=\frac{\rho_{j}}{2}\left[r^{* 2}\left(\Omega^{*}\left(t^{*}\right)\right)^{2}+\overline{\left(V_{j}^{* b}\right)^{2}}\right]+C_{j}
\end{aligned}
$$

the functions $\bar{F}_{j}$ and $\bar{G}_{j}$ are:

$$
\begin{aligned}
& \bar{F}_{j}=\frac{\Omega_{m}}{\sigma_{j}}\left[\frac{\sinh \left(\sigma_{j}\right)+\sin \left(\sigma_{j}\right)}{\cos \left(\sigma_{j}\right)+\cosh \left(\sigma_{j}\right)}-\sigma_{j}\right] \\
& \bar{G}_{j}=\frac{\Omega_{m}}{\sigma_{j}}\left[\frac{\sinh \left(\sigma_{j}\right)-\sin \left(\sigma_{j}\right)}{\cos \left(\sigma_{j}\right)+\cosh \left(\sigma_{j}\right)}\right]
\end{aligned}
$$

whith $\sigma_{j}=\sqrt{\frac{\omega e^{2}}{2 \nu_{j}}}$ is the frequency number.

The pressure jump satisfies the Laplace-Young equation, $\bar{P}_{1}^{* b}-\bar{P}_{2}^{* b}=\frac{\gamma}{R}$ where $\gamma$ is the surface tension.

\subsection{Linear stability}

We assume that the base state is disturbed so that the velocity and the pressure fields in the perturbed state are:

$$
\begin{gathered}
\mathbf{V}_{j}^{*}=\overline{\mathbf{V}}_{j}^{* b}+\mathbf{v}_{j}^{*}\left(u^{*}\left(r^{*}, \theta, z^{*}, t^{*}\right), v^{*}\left(r^{*}, \theta, z^{*}, t^{*}\right)\right) \\
P_{j}^{*}=\bar{P}_{j}^{* b}+p_{j}^{*}\left(r^{*}, \theta, t^{*}\right)
\end{gathered}
$$

To investigate the dynamical evolution of the interface, we describe the dimensional instantaneous interface in polar coordinates as $r^{*}=R+\xi^{*}\left(\theta, t^{*}\right)$, where $\xi^{*}\left(\theta, t^{*}\right)$ is an infinitesimal dimensional perturbation of the circular interface. Hereafter, we seek the solution of the linear problem in terms of normal modes:

$$
\begin{aligned}
{\left[\bar{u}_{j}, \bar{v}_{j}, p_{j}\right] } & =\left[\tilde{u}_{j}, \tilde{v}_{j}, \tilde{p}_{j}\right] \exp (i n \theta) \\
\bar{\xi}(\theta, t) & =\xi_{n}(t) \exp (i n \theta)
\end{aligned}
$$

with $i^{2}=-1, n$ is the azimuthal wave number and $n \geq 1$. To complete the set of equations, we provide the dynamic boundary condition given by:

$\left(\bar{P}_{1}^{* b}+p_{1}^{*}\right)-\left(\bar{P}_{2}^{* b}+p_{2}^{*}\right)+\mu_{2} \frac{\partial \bar{u}_{2}^{*}}{\partial r^{*}}-\mu_{1} \frac{\partial \bar{u}_{1}^{*}}{\partial r^{*}}=\gamma \nabla \cdot \mathbf{n}$

with $\nabla . \mathbf{n}=\frac{1}{R}\left[1-\left(1-n^{2}\right) \frac{\xi^{*}\left(t^{*}\right)}{R}\right]$. Hereafter, the total pressure is linearized, at $r^{*}=R$, as follows

$$
\left(\bar{P}_{j}^{* b}+p_{j}^{*}\right)=\bar{P}_{j}^{* b}(R)+\left.\frac{\partial \bar{P}_{j}^{* b}}{\partial r}\right|_{r=R} \bar{\xi}^{*}(\theta, t)+p_{j}^{*}(R)
$$

The dimensional kinematic condition (using $\omega^{*}$ for time) averaged with respect to the spatial variable $z$ and linearized at the interface is:

$$
A^{-1} \frac{\partial \bar{\xi}_{n}(t)}{\partial t}+i n \frac{\bar{V}_{j}^{b}}{r} \bar{\xi}_{n}(t)=\tilde{u}_{j} \quad \text { at } \quad r=1
$$

$A=\frac{\Omega_{m}}{\omega}$. With the above assumptions, and using the change of variable below:

$$
\begin{aligned}
\xi_{n}(t) & =\bar{\xi}_{n}(t) \exp \left[-\frac{i}{2} \int\left(2(n-1) A \alpha_{1} \bar{V}_{1}^{b}\right.\right. \\
& \left.\left.+2(n+1) A \alpha_{2} \bar{V}_{2}^{b}+\frac{6 i}{\sigma_{1}^{2}} \alpha_{1}+\frac{6 i}{\sigma_{2}^{2}} \alpha_{2}\right) d t\right]
\end{aligned}
$$

where $\alpha_{j}=\frac{\rho_{j}}{\rho_{1}+\rho_{2}}$. Then, the dimensionless form of the parametric differential equation for the amplitude $\bar{\xi}_{n}(t)$ of the interface displacement from its equilibrium position is:

$$
\begin{aligned}
& \frac{d^{2} \bar{\xi}_{n}(\tau)}{d t^{2}}+\frac{\left(1-A_{\rho}\right)}{2 W e_{1}}\left[-\frac{18 W e_{1}}{\left(1-A_{\rho}\right)}\left(\frac{\alpha_{1}}{\sigma_{1}^{2}}+\frac{\alpha_{2}}{\sigma_{2}^{2}}\right)^{2}\right. \\
+ & n\left(n^{2}-1\right)+B_{o}\left(\beta_{1} \cos ^{2}(t)+\beta_{2} \sin ^{2}(t)+\beta_{3} \sin (2 t)\right) \\
+ & \left.i \sqrt{B_{o}}\left(\beta_{4} \cos (t)+\beta_{5} \sin (t)\right)\right] \bar{\xi}_{n}(t)=0
\end{aligned}
$$

with $W e_{1}=\frac{R^{3} \rho_{1} \omega^{2}}{\gamma}$ is the Weber number, $A_{\rho}=\frac{\rho_{2}-\rho_{1}}{\rho_{2}+\rho_{1}}$ is the Atwood number and $B_{o}=\frac{\left(\rho_{1}-\rho_{2}\right) R^{3} \Omega_{m}^{2}}{\gamma}$ is the 
Bond number and

$$
\begin{aligned}
\beta_{1} & =-\frac{1}{A_{\rho}}\left(\Gamma_{1} \bar{F}_{1}^{2}+\Gamma_{2} \bar{F}_{2}^{2}+2 \alpha_{1} \alpha_{2}\left(n^{2}-1\right) \bar{F}_{1} \bar{F}_{2}\right) \\
& -n+\frac{1}{A_{\rho}} n\left(\alpha_{1} V_{F 1}-\alpha_{2} V_{F 2}\right) \\
\beta_{2} & =-\frac{1}{A_{\rho}}\left(\Gamma_{1} \bar{G}_{1}^{2}+\Gamma_{2} \bar{G}_{2}^{2}+2 \alpha_{1} \alpha_{2}\left(n^{2}-1\right) \bar{G}_{1} \bar{G}_{2}\right) \\
& +\frac{1}{A_{\rho}} n\left(\alpha_{1} V_{G 1}-\alpha_{2} V_{G 2}\right) \\
\beta_{3} & =-\frac{1}{A_{\rho}}\left(\Gamma_{1} \bar{F}_{1} \bar{G}_{1}+\Gamma_{2} \bar{F}_{2} \bar{G}_{2}+\alpha_{1} \alpha_{2}\left(n^{2}-1\right)\right. \\
\beta_{4} & =\sqrt{\frac{2 W e_{1}}{-A r(1-A r)}}\left(\Gamma_{3} \bar{F}_{1}+\Gamma_{4} \bar{F}_{2}\right. \\
& \left.+i\left(\alpha_{1} \bar{G}_{1}-\alpha_{2} \bar{G}_{2}\right)\right) \\
\beta_{5} & =\sqrt{\frac{2 W e_{1}}{-A r(1-A r)}}\left(\Gamma_{3} \bar{G}_{1}+\Gamma_{4} \bar{G}_{2}\right. \\
& \left.+i\left(\alpha_{2} \bar{F}_{2}-\alpha_{1} \bar{F}_{1}\right)\right)
\end{aligned}
$$

where $V_{F j}=\left(\Gamma_{5 j}+\Gamma_{6 j}\right) X^{-1}, V_{G j}=\left(\Gamma_{7 j}+\Gamma_{8 j}\right) X^{-1}$, $V_{F G j}=\Gamma_{9 j} X^{-1}$ and $X=4 \sigma_{j}\left(\cosh \left(\sigma_{j}\right)+\cos \left(\sigma_{j}\right)\right)^{2}$. The coefficients $\Gamma_{1}, \Gamma_{2}, \Gamma_{3}, \Gamma_{4}, \Gamma_{5 j}, \Gamma_{6 j}, \Gamma_{7 j}, \Gamma_{8 j}$ and $\Gamma_{9 j}$ are in function of the frequency number $\sigma_{j}, \alpha_{j}$ and the wave number $n$.

\section{Results and Discussion}

Figures $2(a)$ and $(b)$ depict the evolution of the Bond number, $B_{o}$, versus the wave number, $n$, for the waterair interface $\left(\gamma=0.072 \mathrm{~N} / \mathrm{m}\right.$ and $\left.A_{\rho}=-0.9\right)$ and for different values of the dimensional frequency of the oscillations $\omega^{*}$. It is worth noting from results that, the interface is unstable to the parametric instability, unlike in [7-8] where the interface can be linearly unstable to the parametric instability and and to the Rayleigh-Taylor instability due to the centrifugal forces via the constant

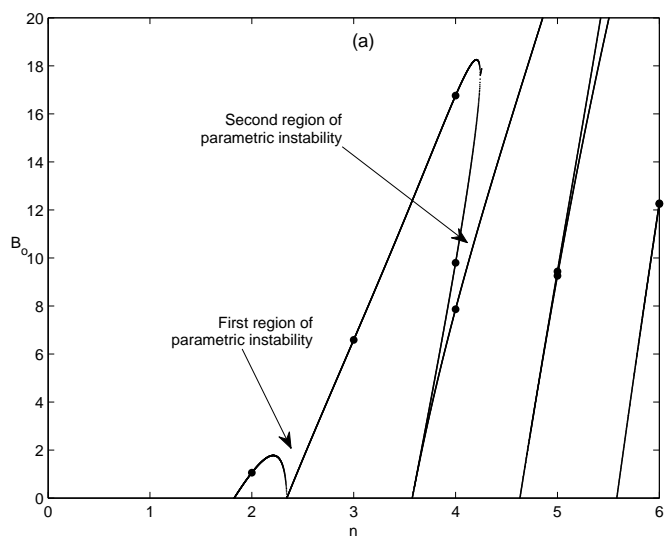

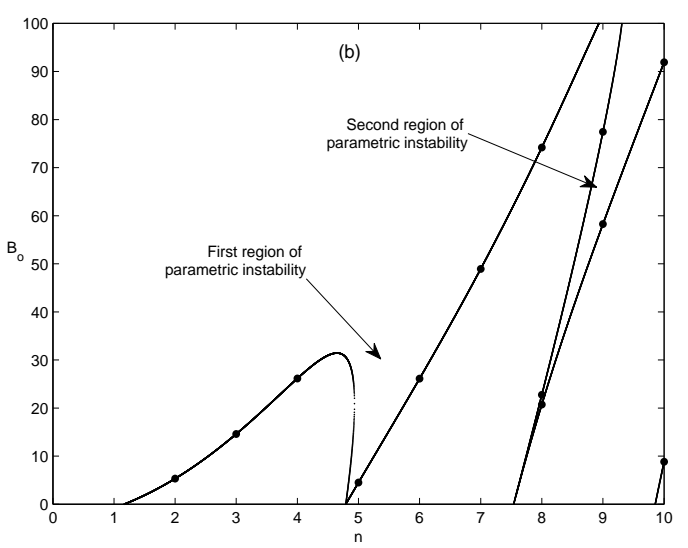

Figure 2: Marginal stability curves, $B_{o}(n)$ for $A_{\rho}=$ -0.9 and for different Weber number and frequency number: (a) $W e_{1}=10, \sigma_{1}=5.985, \sigma_{2}=1.545$ and (b) $W e_{1}=100, \sigma_{1}=18.92, \sigma_{2}=4.88$.

term of rotation. By inspecting this figures, two important main conclusions can be drawn. First, an increase of $\omega^{*}$ which corresponds to increasing the Weber number, $W e_{1}$, as well as the frequency numbers $\sigma_{1}$ and $\sigma_{2}$, tends to shift the resonance zones corresponding to the parametric instability to the right gradually. Indeed, the most unstable wave numbers of the resonances increase significantly with frequency.

\section{Conclusion}

In this investigation, we have performed a linear stability analysis of an interface between two viscous immiscible fluids of different densities in an oscillating circular Hele-Shaw cell. The linear problem is reduced to a periodic oscillator governing the evolution of the amplitude of the interface. In this situation, the oscillation of the cell causes a successive parametric resonance modes. In this work, we have focused our analysis on the effect of the frequency on the boundaries of the marginal stability.

\section{References}

[1] P. G. Saffman and G. I. Taylor, Proc. R. Soc. London, Ser. A 245, 312 (1958) .

[2] L. W. Schwartz, Phys. Fluids. 1, 167 (1989).

[3] L. Carrillo, F. X. Magdaleno, J. Casademunt, and J. Ortin, Phys.Rev. E 54, 6260 (1996).

[4] L. Carrillo, J. Soriano, and J. Ortin, Phys. Fluids. 12, 1685 (2000).

[5] J. A. Miranda, Phys. Rev. E62, 2985 (2000).

[6] A. Abidate, S. Aniss, O. Caballina, M. Souhar, Phys. Rev. E, 75, 046307 (2007).

[7] J. Bouchgl, S. Aniss, M. souhar, A. Hifdi, MATEC Web of conferences 16, 09004 (2014)

[8] Pedro. H. A. Anjos, Victor. M. M. Alvarez, Jos. A. Miranda, Phys. Rev. Fluids 2, 124003 (2017). 